\title{
ENHANCED CLUSTER HEAD MANAGEMENT IN LARGE SCALE WIRELESS SENSOR NETWORK USING PARTICLE SWARM OPTIMIZATION (PSO) ON THE BASIS OF DISTANCE, DENSITY \& ENERGY
}

\author{
Shishir Rastogi \\ School of Computer Applications, Babu Banarasi Das University, Lucknow, INDIA \\ Neeta Rastogi \\ Department of Computer Science and Engineering, \\ Babu Banarasi Das National Institute of Technology \& Management, Lucknow, INDIA

\section{Manuj Darbari} \\ Department of Computer Science and Engineering, \\ School of Engineering, Babu Banarasi Das University, Lucknow, INDIA
}

\begin{abstract}
Wireless Sensor Networks (WSNs) are utilized for a plethora of applications such as weather forecasting, monitoring systems, surveillance, and so on. The critical issues of the WSN are energy constraints, limited memory, and computation time. This spectrum of criticality takes a deep dive with large-scale WSNs. In such scenario, the network lifetime has to be efficiently utilized with the available resources by organizing into clusters. Even though the technique of clustering has proven to be highly effective in minimizing the energy, the tradition cluster based WSNs, the protocol overhead is high for Cluster Heads (CHs) as it receives and aggregates the data from its cluster members. Therefore, efficient management of $\mathrm{CH}$ along with routing behavior is vital in prolonging the network lifetime. In this paper, an enhanced CH-Management technique is proposed which efficiently elects its $\mathrm{CH}$ using Particle Swarm Optimization (PSO), hereafter referred to as PSO_DDE. The PSO_DDE approach considers various parameters such as within-cluster distance between nodes (intra-cluster distance), neighbor density, and residual energy of nodes for the best candidate selection of $\mathrm{CH}$. Also, the cluster formation is defined by the k-means based on the Euclidian distance. The PSO_DDE approach is integrated with the Dynamic Source Routing (DSR) for efficiently traversing the data packet to the sink node. The performance metrics are compared with the existing approaches using NS-2 simulator, and the proposed approach shows superiority of results.
\end{abstract}


Enhanced Cluster Head Management in Large Scale Wireless Sensor Network Using Particle Swarm Optimization (Pso) On the Basis of Distance, Density \& Energy

Keywords: Cluster Management, Cluster Head Selection, Data Aggregation, k-means, PSO, DSR.

Cite this Article: Shishir Rastogi, Neeta Rastogi And Manuj Darbari Enhanced Cluster Head Management in Large Scale Wireless Sensor Network Using Particle Swarm Optimization (Pso) On the Basis of Distance, Density \& Energy.International Journal of Computer Engineering and Technology, 10(1), 2019, pp.203-217 $\mathrm{http}: / /$ iaeme.com/Home/issue/IJCET?Volume $=10 \&$ Issue $=1$

\section{INTRODUCTION}

Wireless sensor networks contain several randomly distributed sensor nodes over a physical geography that collects data from the surrounding environment. As the location of sensor nodes is not needed to be determined in advance, they can be deployed over an area which may be inaccessible [1]. Wireless sensor nodes are generally operated on internal power source like batteries often in isolated environments where batteries cannot be recharged, and battery outage can cause sensor node's disconnection from the network. Sensor nodes consume most of their energy in communicating with other nodes [2] [7]. It is pertinent to optimize the data communications among sensor nodes for effective and efficient use of resources, which are albeit limited, to enhance network lifetime [7].

Clustering is an approach to group nodes and organize them into small clusters. As a convention, clustering is being used for establishing a network hierarchically. The aim is to achieve Energy efficient and robust way of transmission within the network [3], [7]. Each cluster has a cluster head liable for forwarding collected or aggregated data from member sensor nodes to the base station (BS) directly or via a sequence of CHs through multi-hop. Clustering offers various advantages over flat architecture like reducing inter-node communication, increasing network scalability, better bandwidth management and allowing sensor nodes to go into sleep mode for the spells of time resulting in energy savings [3], [7]. CH selection is an important issue of any cluster-based approach that directly affects the performance of network. For selecting the $\mathrm{CH}$, number of criteria are used as selection based on single criteria (e.g., residual energy) may not lead to optimal performance because the selected $\mathrm{CH}$ may not be a good choice [4]. Various parameters like residual energy, distance from other nodes and cluster centroid, etc. are accounted for in selection of optimum $\mathrm{CH}$ [7]. In a general clustering algorithm, many nodes in a network are chosen as the cluster heads (CHs) and other nodes are considered as cluster members (CMs), forming connections with the $\mathrm{CHs}$. A $\mathrm{CH}$ receives data from its CMs. A WSN involves a number of sensor nodes in set, not controlled by any external sources. WSNs are self-organized and they possess communication links to collectively finish the tasks without any coordination done by any central server. Extracting accurate data is another complex issue in sensor networks. WSNs comprise a large number of sensor nodes, small autonomous wireless devices, powered by an internal small battery. The sensor nodes consume energy in sensing, communication, and computation of data. Thus, designing an energy efficient routing scheme for collection and aggregation of sensor data to attain a high delivery ratio with an enhanced lifetime of the network is a challenge.

In this pwaper, an improved CH-Management technique based on Particle Swarm Optimization (PSO), referred to as PSO_DDE, is being proposed to enhance the network lifetime. PSO_DDE is employed to determine the optimal region quickly. It updates the populace of nodes as per the fitness information so that better solutions can be expected for the operation of WSN. 
The salient features of the proposed method are

- K-means approach used for Cluster Formation and PSO (Particle Swarm Optimization) for Cluster Head Management

- PSO approach is integrated with the Dynamic Source Routing (DSR) for efficient data communication of the data packets from the sensor nodes to the sink node.

- The overall objective is to lessen the amount of data transmission and reduce energy dissipation in communication

Rest of the paper is organized as follows. Section 2 presents related work. Section 3 describes the PSO method in brief, the proposed method of cluster management using k-means for clustering based on Euclidian distance of node from the centroid of the cluster followed by PSO for cluster head selection and efficient transmission using DSR protocol. Section 4 presents the results and discussions. The paper is concluded in Section 5 with future directions.

\section{LITERATURE REVIEW}

Jerbi, W. et al. (2016) considered the consequences of isolated nodes that are away from the cluster head $(\mathrm{CH})$ or if $\mathrm{CH}$ is not within reach from these nodes. They proposed Orphan Low Energy Adaptive Clustering Hierarchy $(\mathrm{O}-\mathrm{LEACH})$, a routing protocol that incorporates the orphan nodes. Their contribution was to elect a cluster head that has sufficient energy for a better now to coordinate with these member nodes and maintain the full coverage for applications which requires of useful data for the entire area to be covered. They proved that the results were better than LEACH protocol when parameters like connectivity rate, energy, scalability, and coverage were taken into account [6].

Farman, H., et al. (2017) employed a multi-decision criteria tool called Analytical Network Process (ANP) to choose the most appropriate node as a $\mathrm{CH}$ among available nodes. ANP was introduced to solve dependency issues for various elements and the feedback between network layers which was missed out in its previous version called Analytical Hierarchy Process (AHP) [7].

Deepa. O and Suguna. J (2017) proposed Optimized QoS-based Clustering with Multipath Routing Protocol (OQoS-CMRP) for WSNs to decrease the consumption of energy in the destination coverage area by employing the Modified PSO based clustering algorithm to form clusters, to choose cluster heads in the sink coverage area and to solve the problems regarding energy hole. The Single Sink-All Destination algorithm was used to find near-optimal multihop communication path from sink to sensors for selecting the next hop neighbor nodes. The Round-robin Paths Selection algorithm was applied for transferring data to the destination. The results proved that OQoS-CMRP attained efficient communication of data with quite enough energy conservation [8].

Yi Gu and Qishi Wu (2009) investigated the problem of selecting nodes in a pre-deployed sensor network to minimize the total energy needed for data gathering. They derived a formula analytically to optimize cluster heads under a uniform distribution of node. Distance-based Crowdedness Clustering algorithm was proposed to determine the cluster heads in sensor networks under the general distribution of node [9].

Rasheed et al., (2005) proposed an enhanced realistic clustering scheme to form a hierarchical control structure for very large-scale ad hoc networks. The proposed clustering scheme was distributed and enabled the formation of stable and scalable clusters [10].

Ahmad et al., (2018) depicted the honey bee algorithm to elect a cluster headset from existing nodes in the network and form clusters based on cluster heads. The issue was first applied to the dynamic optimization problem, and a honey bee algorithm was employed to choose the most appropriate cluster heads in the network [11]. 
Zhoujing Ye et al., (2017) proposed Strip Clustering Scheme (SCS) to substitute the Conventional Scheme (CS). This method incorporates region division, selection of $\mathrm{CH}$, link establishment, data fusion, and transmission. Adopting SCS can decrease total energy consumption in the network and several redundant data by the data fusion process. Extra energy of head nodes was obtained by summing up the number of the battery within the head nodes or utilizing renewable energy technologies [12].

Rao, P. S. et al., (2017) proposed a cluster head selection algorithm especially for energy efficiency which was based on PSO-ECHS. The algorithm was formulated with a method of the fitness function and particle encoding. The parameters like destination-distance, intracluster distance, and sensor nodes' residual energy were considered to attain efficient energy. The experimental outcomes showed that their proposed algorithm performed better than the existing algorithms regarding network lifetime, total energy consumption, and the packets of data received by the base station [13].

Rajagopal, A et al., (2018) in their work adopted the Bacterial Foraging Optimization (BFO) for cluster head $\mathrm{CH}$ selection such that the objectives such as improved cluster formation, decrement in packet delivery ratio, increment in network lifetime and decrement in end to end transmission delay can be achieved. Also, a novel Hybrid algorithm using BFO-Bee swarm Optimization (BSO) was applied to analyze the number of clustered formed, packet drop ratio, an end to end transmission delay, and lifetime [14].

Singh, S. S (2002) surveyed the application of particle swarm optimization (PSO) in WSN over a different type of clustering-based algorithm techniques like LEACH, LEACH-C, PEGASIS, etc. Several cluster-based algorithms were applied. They determined all type of PSO based algorithm, their application, and limitation over present methodologies to overcome the issues of low energy and coverage of sensor range. By this application, the efficiency of WSN was increased, and also the power delay of the information was reduced, and the range of sensor nodes was increased [15].

Ali S., Fathima, K., \& Sumitha, T. (2014) analyzed that major challenge in WSNs was to increase the network lifetime. One of the best energy efficient protocol, LEACH was used to attain less energy consumption and to expand the lifetime of WSN. If the BS is too far from the $\mathrm{CH}$, more energy is consumed which in turn decrease the lifetime of WSN. To overcome these, PSO was implemented with LEACH protocol to attain maximum lifetime, extend the scalable and energy efficiency [16]. 


\section{PROPOSED METHODOLOGY}

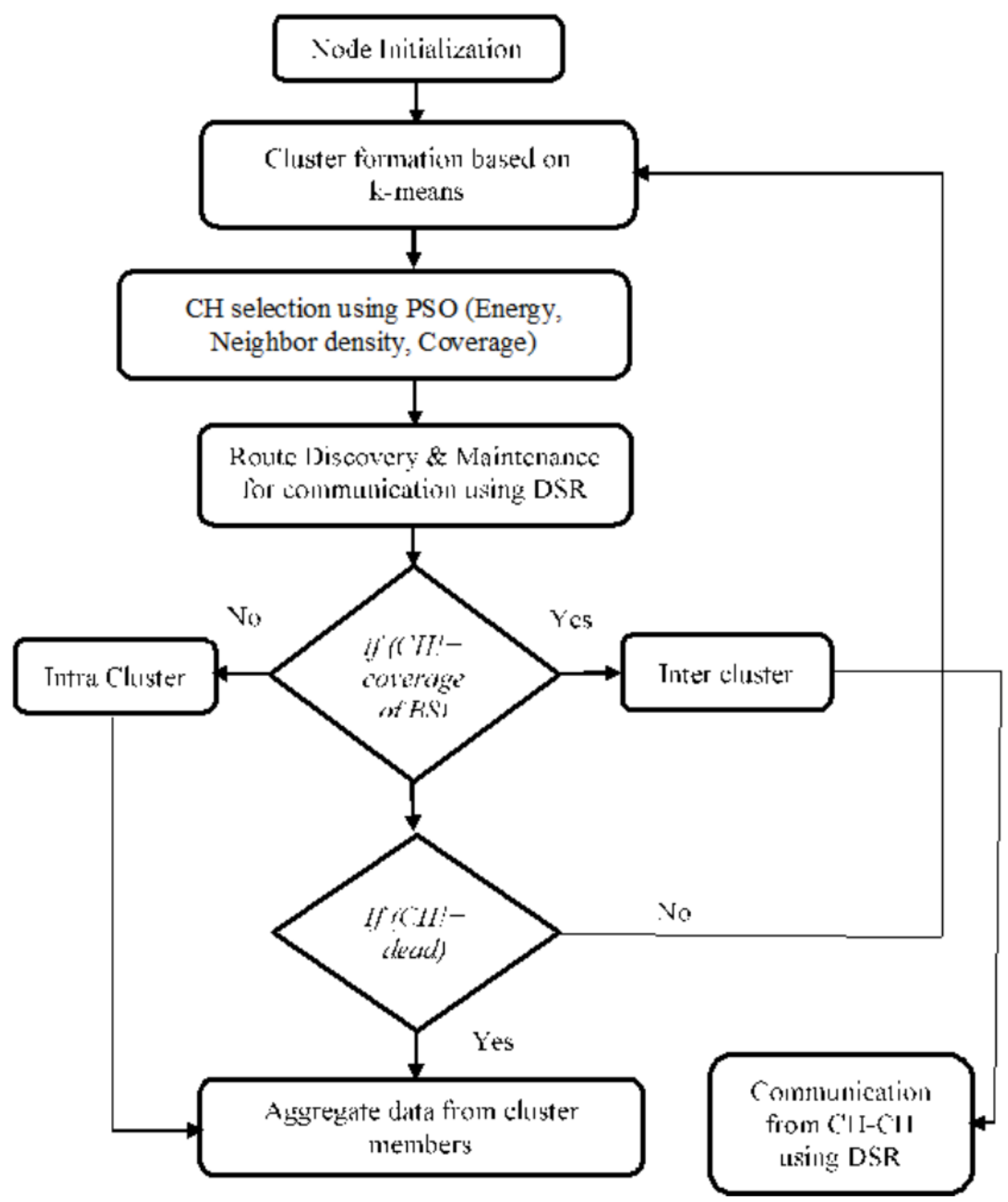

Figure 1 Flow Chart of Proposed Methodology

In the proposed methodology as represented through flow chart in Figure 1, firstly, the nodes are initialized. A cluster with members is formed based on k-means. Among the members of the cluster, a cluster head has to be selected. This is done by using PSO approach. Selection of cluster heads is performed by considering the parameters like the energy of the nodes, neighbor density, and area coverage of the nodes. An appropriate and shortest path or route is determined by using the DSR protocol, and it also helps in maintaining the communication between the nodes efficiently. If the cluster head is located within the coverage of base station, then the packets are traversed to the intracluster and aggregation of data from cluster members is done. In the case of cluster head located out of the coverage of base station, the packets are 
Enhanced Cluster Head Management in Large Scale Wireless Sensor Network Using Particle Swarm Optimization (Pso) On the Basis of Distance, Density \& Energy

transmitted to the intercluster. Then the communication between Cluster Heads is done by using DSR. Aggregation of data happens only when the cluster head is not dead. If the cluster head is found dead, a new cluster is then formed. The process is repeated for efficient and quick communication between clusters in a large-scale wireless sensor network.

\subsection{PSO Algorithm}

Particle Swarm Optimization is a heuristic global optimization method. The concepts of PSO incorporate velocity changes (acceleration) of each particle towards its pbest and gbest (global version of PSO) positions per time step. Acceleration is measured with individual random numbers for determining acceleration towards pbest and gbest positions. In addition to pbest, there is also a local version of PSO called lbest, in which each particle keeps tracking the best solution.

The Step-wise process for determining the gbest PSO is as follows:

1. Initialize a collection of particles with random positions and velocities on $d$ dimensions in the problem space.

2. For each particle, determine the desired optimization fitness function in $d$ variables.

3. Compare the fitness function with pbest of the particles. If the presently obtained value is greater than pbest, then set pbest value equal to the currently obtained value, and the pbest location equal to the current location in $d$-dimensional space.

4. Compare fitness function with the population's overall previously achieved best values. If the presently obtained value is greater than gbest, then reset gbest to the currently obtained particle's array index and value.

5. Change the position and velocity of the particle as given in equations (1) and (2) respectively:

$$
\begin{gathered}
v_{i d}=v_{i d}+c_{1} * \operatorname{Rand}() *\left(p_{l d}-x_{i d}\right)+c_{2} * \operatorname{Rand}() *\left(p_{g d}-x_{i d}\right) \\
x_{i d}=x_{i d}+v_{i d}
\end{gathered}
$$

6. Go to step (2) until a criterion is met usually a sufficient and better fitness or several numbers of iterations.

The velocity of particles in every individual dimension is maintained at maximum velocity $V \max$. Vmax measures the resolution, with which area between the current and the target (best so far) locations are searched. If Vmax is much high, particles can provide good solutions. If Vmax is much small, then, particles cannot inquire enough beyond locally good regions. They may be trapped in local optima, incapable of moving far enough to reach a better location in the problem space.

The acceleration constants $c_{1}$ and $c_{2}$ in equation (1) denote the weighting of the stochastic acceleration terms that transfers each particle toward pbest and gbest positions. Here, particles only their data and their neighbors' bests, instead of the entire group. Instead of transferring toward a kind of stochastic average of pbest and gbest (the best location of the entire group), particles transfer toward points defined by pbest and lbest, which is the particle's index with the best estimation. If the neighborhood size is equal to two, for instance, the particle (i) compares its fitness value with particle (i-1) and particle (i+1). Neighbors are called topological neighbors. For the neighborhood version, the only alternative to the process defined in the six steps before is the substitution of $p_{l d}$, which is the location of the neighborhood best, for $p_{g d}$, the global best, in equation (1). Early experience (trial and error) led to neighborhood sizes of about 15 percent of the population size being used for many applications. So, for a group of 40 particles, a neighborhood of six, or three topological neighbors on each side, is not unusual. 


\subsection{Proposed PSO_DDE Method for Cluster Management using k-means Clustering and PSO for cluster head selection}

Step 1: $\quad$ Node Deployment in the given area (A)

Step 2: $\quad$ Node Initialization initiated by Base Station (BS) $€$ vector $V$

Step 3: $\quad$ BS Computes Cluster formation by initializing the ' $k$ ' number of cluster groups to be formed from step 4, step 5, and step 6 based on K-means.

Step 4: $\quad$ BS selects ' $k$ ' number of centroids based on random position from the assumed ' $\mathrm{k}$ ' cluster groups.

Step 5: Calculate the Euclidian distance from each node to all centroids and assign it to centroid nearest to it and ' $\mathrm{k}$ ' clusters are formed.

Step 6: Suppose $\mathrm{n}$ nodes are given such that each one of them belongs to Rd. The problem of finding the minimum variance clustering of these nodes into $\mathrm{k}$ clusters is that of finding the ' $\mathrm{k}$ ' centroids

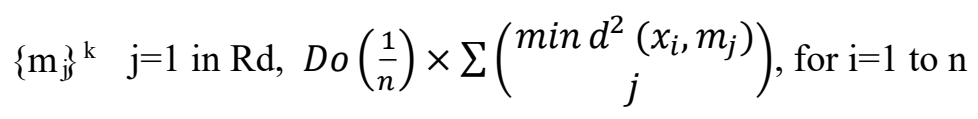

Step 7: With the change in the position of the centroid repeat step 5.

Step 8: $\quad$ BS selects the Cluster Head $(\mathrm{CH})$ by integrating with PSO by calculating the fitness parameters (Energy, Neighbor density, Coverage) and Best node with high fitness value is elected as $\mathrm{CH}$.

Step 9: BS initiates DSR protocol for Route Discovery and Maintenance for communication.

Step 10: If $\mathrm{CH}$ ! = within the spectrum coverage of BS then BS initiates Inter cluster communication using DSR

Else BS initiates Intra cluster communication using DSR

Step 13: If $\mathrm{CH}$ ! = dead then $\mathrm{CH}$ Aggregates data from cluster members and forward to BS

Else Go to Step 3 and repeat the process

\subsubsection{Cluster Head Selection using PSO approach}

A network's performance relies on cluster head selection, as it is much essential for every energy efficient protocol in WSN. A poor selection can lead to network instability and lowering network lifetime as it has a direct impact on the entire network. As the $\mathrm{CH}$ is responsible for data gathering and forwarding, it is important to select the optimum node as $\mathrm{CH}$ that can extend the network stability and lifetime. Here, we have considered three distinct parameters 1) Distance from neighboring nodes within a cluster 2) Density and 3) Energy to be carried out by each node. Each node's movement is affected by its best-known local position, but it is also directed towards the best-known positions available in the search-range, which are recorded and updated as best positions and detected by other nodes [17]. As energy is the major concern in WSN, PSO provides us with an optimal selection of cluster heads, i.e., the scarce distribution of cluster heads over the network [18]. Energy dissipation includes data packet processing, transmitting, receiving and fusing in the transceiver. It includes mobile nodes, and their positions can be found using GPS. A sensor node $n$ is decided based on threshold formula which is given as

$$
\begin{aligned}
& T_{c h 1}(n, r)=\left\{\frac{P N}{N_{\text {active }}} \text {, if } \frac{P N}{N_{\text {active }}}<1\right. \\
& T_{\text {ch } 1}(n, r)=\left\{1, \text { if } \frac{P N}{N_{\text {active }}} \geq 1\right.
\end{aligned}
$$


Cluster heads are selected by considering the optimized selection and node energy equilibrium. The data is received and collected by Master Cluster Head (MCH), and then it is forwarded to Vice Cluster Head ( $\mathrm{VCH})$. $\mathrm{VCH}$ receives the collected data and transmits it directly to the destination. $\mathrm{MCH}$ is the current global best of a cluster and $\mathrm{VCH}$ is the previous global best. Since the $\mathrm{MCH}$ does not interact directly with the sink, energy is saved resulting in a prolonged lifetime of the network.

The algorithm can be split into three logical parts. In the first part, the clusters are formed. In the second part, the cluster-heads are selected in each cluster. In the third part, every node in the network is assigned to CHs. Firstly, PSO optimization is performed. It is used to find a line partitioning the nodes into two regions comprising both the same number of nodes (for M even) and the same number of available CHs. Equal division of the available cluster-heads is required to ensure that subsequent divisions of resulting groups will still contain candidate cluster-heads.

The PSO particle is three dimensional and has the following form,

$$
\bar{P}=(x, y, \theta)
$$
line.

where $x, y$ is a point on the line partitioning the two regions and $\theta$ is the orientation of the

The fitness function is given in equation (6):

$$
\text { Fitness }=\left(a_{1}-f_{1} A\right)^{2}+\left(a_{2}-f_{2} A\right)^{2}+\left(c_{1}-f_{1} N\right)^{2}+\left(c_{2}-f_{2} N\right)^{2}
$$

Two regions are formed after the partitioning. These are referred to as region 1 and region 2. The subscripts in the fitness function refer to the regions. In the fitness function, $\mathrm{N}$ denotes a group of nodes, where $a$ nodes are available for the $\mathrm{CH}$ role. $f$ is the available $\mathrm{CHs}$ in region $i$ and $c_{i}$ is the number of nodes in region $i$.

$$
f_{i}=\frac{M_{i}}{M}
$$

where $M_{i}$ is the number of required clusters in the region $i$. If $M$ is even, then $M_{1}=M_{2}$, and $f_{1}=f_{2}=1 / 2$. This formulation allows us to handle the general case where $M$ might also be odd. The fitness represents the deviation from our prescribed optimum that we balance the nodes and available $\mathrm{CHs}$ in each region. A perfect partitioning of the nodes will cause fitness to become zero [19].

Our PSO optimization is a real number optimization and as such will respond best to continuous functions. By inspection, one can verify that by varying $x, y$ and $\theta$, the counts of nodes and available cluster-heads in each region created by the PSO particle line, will vary smoothly, albeit discretely.

This is a good indication that the PSO approach will respond favorably to our selection of node and fitness. At the end of the recursive optimization, we are left with clusters of nodes, where there exists at least one available $\mathrm{CH}$ in each region. The algorithm also involves selecting the node in each region, from those that are available to be cluster-heads that will be the assigned cluster-head. This is not a hard problem. The cluster is fixed, and we are looking for just one cluster-head. We are looking for the one node, from the available CHs group, which will decrease the mean distance between a non- $\mathrm{CH}$ node and a $\mathrm{CH}$. This part of the algorithm is performed by calculating the mean distance for every possible cluster-head and selecting as cluster-head which produced the minimum mean distance. The sensors are uniformly deployed in the sensing region. Moreover, every sensor node is distributed as per 2-dimensional Poisson point process with an expected area density of the cluster. 


\subsubsection{Efficient transmission using DSR protocol}

The BS initiates DSR protocol for discovering the route and for communication maintenance. DSR is an efficient reactive protocol specifically used in multi-hop wireless ad hoc networks consisting mobile nodes. DSR never require administration or infrastructure of an existing network. This means that DSR permits the network to be self-configuring and self-organizing. DSR is an imprudent routing protocol that utilizes source routing to send packets in which the source must know the complete hop sequence to the destination. In DSR the source finds the whole hops sequence that every packet should traverse. This requires that the sequence of hops is involved in the header of each packet. A negative impact of this is the routing overhead which every packet carries. DSR utilizes source routes to control the packets forwarding via the network.

The key benefit of a source routing design is that intermediate nodes do not require to maintain global routing data updates since the packets themselves already consist of all the routing decisions. This fact, coupled with the on-demand nature of the protocol, dramatically decreases the overhead of the protocol by rejecting the requirement for the periodic route advertisement and neighboring packets detection that is present in other protocols. Beyond this, the source route on each packet describes a path via the network. Hence, if there are no additional packets, every node overhears a source route by learning a way to reach all nodes listed on the route.

The protocol involves two main mechanisms of Route Discovery and Route Maintenance, which work jointly together to permit nodes to discover and maintain routes to arbitrary sinks. The protocol operates on-demand, permitting the routing packet overhead of DSR to scale automatically to only that is needed to react to the route's changes which are presently in use. Route Discovery determines the optimum path for a transmission between a given source and destination. Route Maintenance affirms that the transmission path remains optimum and loopfree as conditions of the network change, even if route changing is essential at the time of transmission.

Route Discovery is a process which establishes a source route to D when a node S (Source) wants to send a packet to a destination node $\mathrm{D}$ (Destination). This is done when $\mathrm{S}$ tries to send a packet to $\mathrm{D}$ and when the route to $\mathrm{D}$ is not known. Route Maintenance is the process by which node $\mathrm{S}$ can detect while utilizing a source route to $\mathrm{D}$ if the network topology has modified such that it can no longer utilize its route to $\mathrm{D}$ since a connection along the route no longer is utilized for data transmission. During Route Maintenance, if a source route is disconnected, S can try to utilize any other route it happens to know to D, or it can find out the Route Discovery again to determine a new route for the upcoming packets to $\mathrm{D}$. Route Maintenance for this route is utilized only when $\mathrm{S}$ is actually transmitting packets to $\mathrm{D}$. For example, when node $\mathrm{S}$ wishes to send a packet to $\mathrm{D}$, but it is unaware of the route to $\mathrm{D}$, node $\mathrm{S}$ begins a route discovery. [20]

Source node S distributes Route Request (RREQ) packets (also called query packets) in the network. Each node appends its address in the packet header when forwarding RREQ. A node receiving an RREQ rebroadcasts it exactly once. Destination D sends a Route Reply (RREP) when the first RREQ is received. RREP is sent on a route which is obtained by returning the route affixed to receive RREQ. RREP includes the returned route from $S$ to $D$ on which RREQ was received by node D. Node Son receiving RREP, stores the route included by RREP. When $\mathrm{S}$ sends a data packet to $\mathrm{D}$, the entire route is affixed in the packet header. Hence DSR is also referred to as source routing. Intermediate nodes utilize the source route incorporated in a packet to find out to whom a packet should be sent. Source route size grows with route length. If the next hop link is broken when a data packet is being forwarded, a Route Error (RERR) is generated and propagated backward. RERR contains the failed link information. When $\mathrm{S}$ 
receives RERR, it erases any cached route with the failed link. When forwarding any packet, the node learns a route to all nodes in the source route contained in the packet.

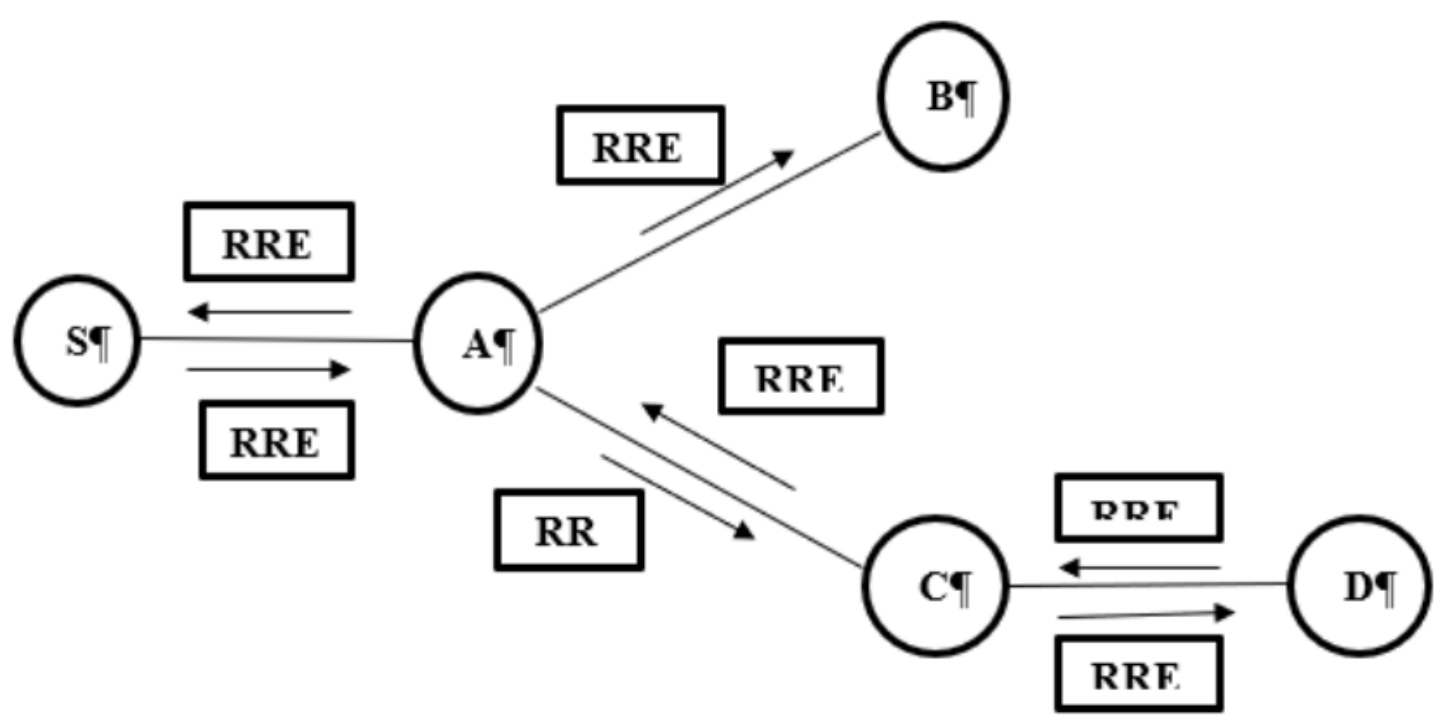

Figure 2 Transmission of Packets using DSR protocol

Benefits of DSR are:

- Source routing: No special method required to eliminate loops.

- On-demand routing: Routes maintained only between nodes which desire to communicate (Reduces overhead of route maintenance).

- Route caching (Storing) can further decrease overhead in route discovery.

- A single route discovery may yield a lot of routes to the node $\mathrm{D}$, due to intermediate nodes replying from local caches. This is applicable when the route is disconnected [21].

We select a route by avoiding the node which is tending to die out. This way we are removing the route which may break early. To save the remaining battery energy, we use a hopby-hop control mechanism in which the nodes that receive an RREQ at the power $P_{r c}$ which transmitted it at $P_{t r}$, calculates the new transmission power $P_{n e w}$ for this receiving node such that this node can communicate with the sender by using this minimum required power $P_{\text {new }}$ using:

$$
P_{\text {new }}=P_{\text {tr }}-P_{r c}+P_{\text {threshold }}+P_{\text {margin }}
$$

where $P_{\text {threshold }}$ is the required threshold power of the receiving node for successful reception of the packet and $P_{m a r g}$ in is the power included to overcome the problem of unstable links due to channel fluctuations. While sending back the RREP, it sends the same power to the sender node, and it uses this power for data packet transmission. The calculated power at each node is stored, and this is the minimum required power for successful transmission and reception. The node rebroadcast the RREQ with maximum power if it is not the destination. The next hope node also does the same procedure, and it will continue up to the destination.

\section{RESULTS AND DISCUSSION}

The proposed method is evaluated regarding coverage, residual energy, and network density. The evaluation process includes network formation, cluster formation, route discovery and maintenance, node communication (either intra or intercommunication) followed by data aggregation. The proposed network scenario is designed and implemented using Network 
Simulator-2. At first, parameters are considered for simulations as shown in table 1. Then throughput, end-end delay, and energy consumption are estimated.

Table 1 Simulation Parameters

\begin{tabular}{|c|c|}
\hline Parameters & Values \\
\hline Number of nodes & 73 \\
\hline Packet Size & 50 \\
\hline Routing Protocol & DSR \\
\hline Simulation Time & $40 \mathrm{~s}$ \\
\hline Dimension of Topography (x, y) & 1186,600 \\
\hline Interface Queue Type & Drop tail / PriQueue \\
\hline Antenna Type & Omni Antenna \\
\hline Application Layer Protocol & UDP \\
\hline Channel & Wireless Channel \\
\hline Radio Propagation Model & 500 Meter \\
\hline Coverage Range & 73 \\
\hline Node Density & K-means \\
\hline Cluster & PSO \\
\hline Route Optimization & Grid Topology \\
\hline Topology & Two \\
\hline
\end{tabular}

In Large-Scale Wireless Sensor Network, initially, a network is formed by a set of nodes. For every node, a unique id is assigned such as n1, n2, n3, n4, n5, n6, n7, and ni. These nodes collectively form a network. Then Cluster is formed using k-means based on Euclidian distance. BS selects the Cluster Head $(\mathrm{CH})$ integrating with PSO by calculating the fitness parameters (Energy, Neighbor density, Coverage). A best node within the cluster with high fitness value is elected as CH. For Route Discovery, BS initiates DSR protocol through broadcast request. After discovering the efficient route, intra communication or intercommunication is established. If $\mathrm{CH}$ is within the coverage of Base Station, Intracluster communication is initiated by BS. If CH is out of coverage from $\mathrm{BS}, \mathrm{CH}$ communicates to $\mathrm{CH}$ of another cluster due to node density in LSWSN. Then, the $\mathrm{CH}$ collects data from all cluster members and forward the collected data to Base Station to save energy consumption of the established network. Data aggregation is shown in Figure 3.

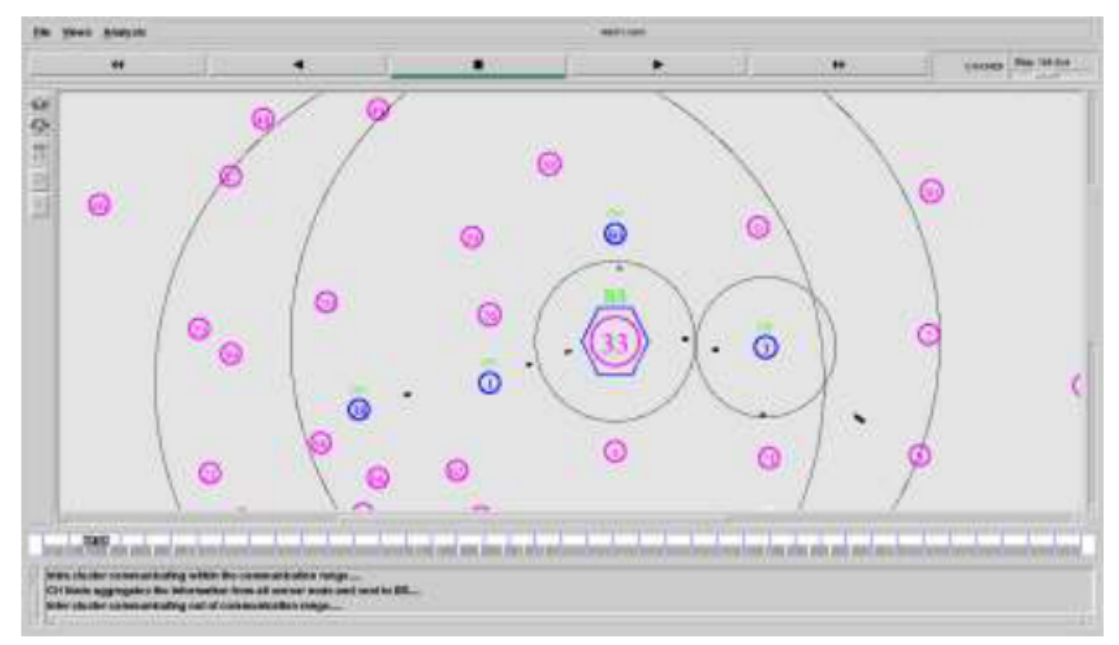

Figure 3 Data Aggregation in Large Scale Wireless Sensor Network 
Enhanced Cluster Head Management in Large Scale Wireless Sensor Network Using Particle Swarm Optimization (Pso) On the Basis of Distance, Density \& Energy

The performance of the proposed method PSO_DDE is compared with the plain DSR based approach of Cluster Management on three parameters: Throughput, Energy Consumption and End-to-end Delay

Throughput is calculated for the PSO_DDE method and DSR approach-based method by using the formula given below,

$$
\text { Throughput }=(\text { received data*8/Data Transmission period })
$$

The obtained throughput values are compared. Figure 4 shows the comparison between the proposed PSO_DDE method and DSR. From the comparison, it has been observed that the proposed PSO_DDE method achieves high throughput than DSR.

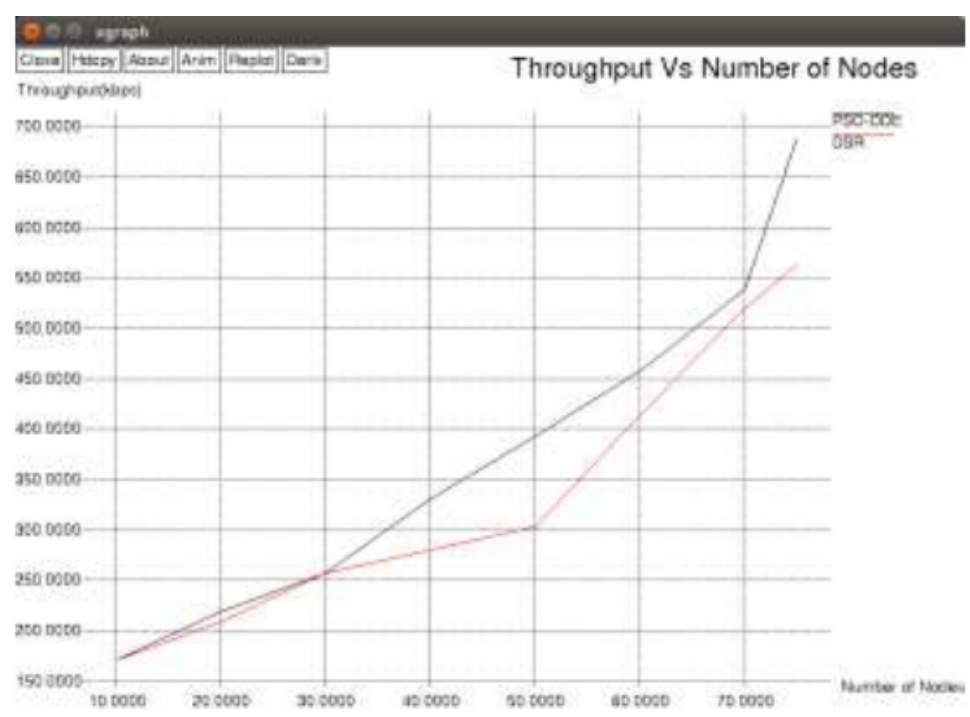

Figure 4 Throughput Analysis

Energy consumed by the nodes is the difference between their initial energy and the final energy. The energy consumed by the nodes in both DSR and PSO_DDE is estimated. The obtained energy consumption values are compared as shown in figure 5 . It is observed that in PSO_DDE, the nodes consume low energy compared to DSR.

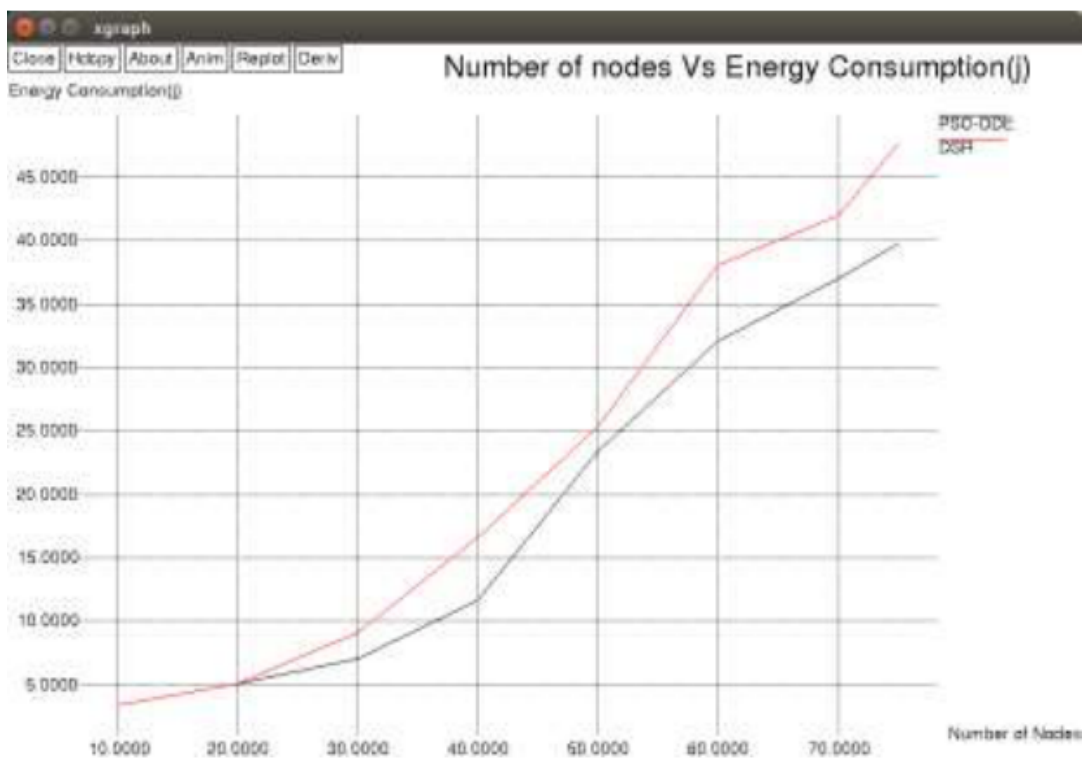

Figure 5 Energy Analysis 
The end-to-end average delay in packet transmission of the proposed PSO_DDE method and the Dynamic Source Routing (DSR) is calculated, and their comparison is shown in figure 6. After comparison, it is observed that the packets are transmitted quickly in the proposed PSO_DDE method when compared to DSR.

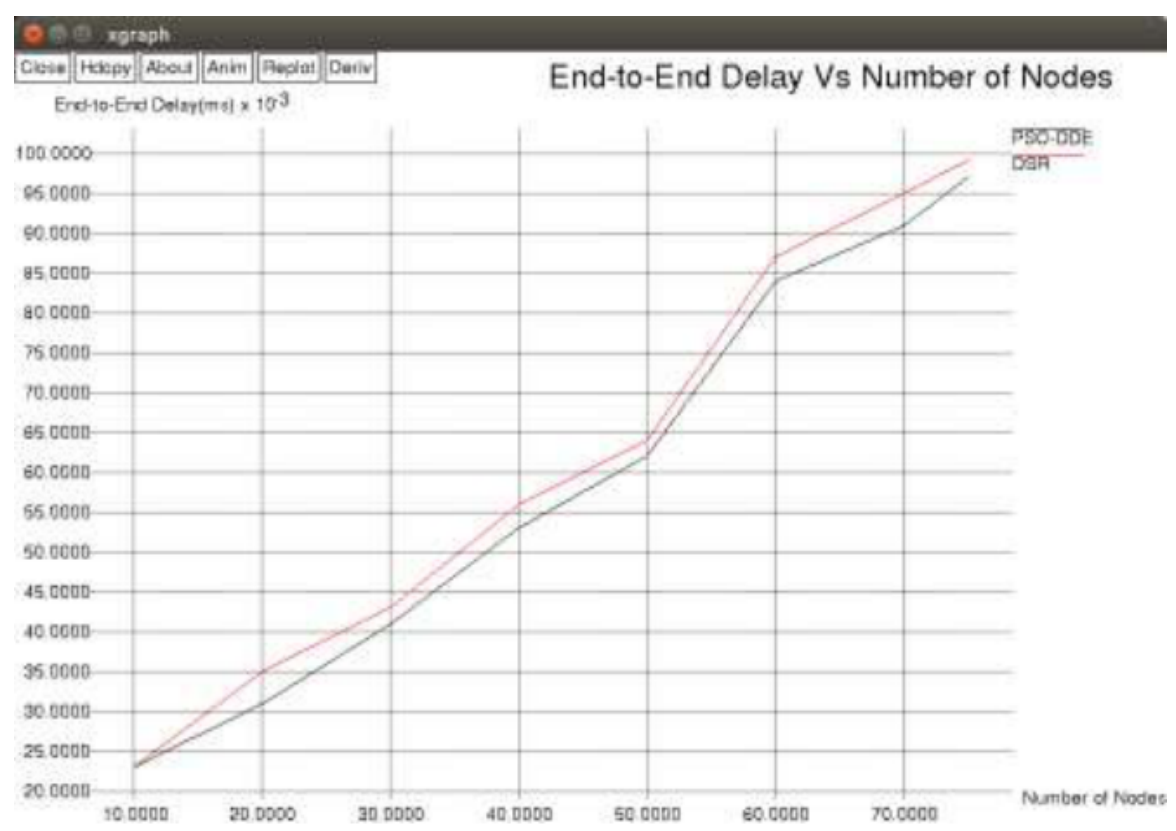

Figure 6 End-to-End Delay Analysis

\section{CONCLUSION}

In the proposed PSO_DDE method, PSO approach was successfully used for Cluster HeadManagement in Large Scale Wireless Sensor Network. The PSO_DDE algorithm considered three parameters such as intra-cluster distance, neighbor density, and residual energy for the best candidate selection of $\mathrm{CH}$. The PSO approach with the Dynamic Source Routing (DSR) efficiently traversed the data packet to the sink node. The performance metrics of PSO_DDE were compared with DSR approach-based method. The simulation results showed that the proposed approach shows a superior performance in terms of Throughput, Energy consumption and End-to-end delay.

\section{REFERENCES}

[1] Batra, P. K. \& Kant, K. LEACH-MAC: A new cluster head selection algorithm for Wireless Sensor Networks. Wireless Networks, 22(1), 2016, pp. 49-60.

[2] Hacioglu, G., Kand, V. F. A., \& Sesli, E. Multi objective clustering for wireless sensor networks. Expert Systems with Applications, 59, 2016, pp. 86-100.

[3] Aslam, N., Phillips, W., Robertson, W., \& Sivakumar, S. A multi-criterion optimization technique for energy efficient cluster formation in wireless sensor networks. Information Fusion, 12(3), 2011, pp. 202-212.

[4] Ghodsypour, S. H., \& O'Brien, C. A decision support system for supplier selection using an integrated analytic hierarchy process and linear programming. International Journal of Production Economics, 56, 1998, pp. 199-212.

[5] Singh, Rajkaran, \& Sharma, Amit. Design Issues and Parameters for Cluster Head Selection in Energy Efficient Wireless Sensor Networks. International Journal of Advanced Research in Computer Science and Software Engineering. 5(10), 2015. 
Enhanced Cluster Head Management in Large Scale Wireless Sensor Network Using Particle Swarm Optimization (Pso) On the Basis of Distance, Density \& Energy

[6] Jerbi, W., Guermazi, A., \& Trabelsi, H. A novel clustering algorithm for coverage a large scale in WSN. arXiv preprint arXiv:1605.03079, 2016

[7] Farman, H., Javed, H., Jan, B., Ahmad, J., Ali, S., Khalil, F. N., \& Khan, M. Analytical network process based optimum cluster head selection in wireless sensor network. PloS One, 12(7), 2017, e0180848.

[8] Deepa, O., \& Suguna, J. An optimized QoS-based clustering with multipath routing protocol for wireless sensor networks. Journal of King Saud University-Computer and Information Sciences, 2017 Computer and Information Sciences (2017), https://doi.org/10.1016/j.jksuci.2017.11.007

[9] Gu, Y., \& Wu, Q. Optimization of cluster heads for energy efficiency in large-scale wireless sensor networks. In International Conference on Ad Hoc Networks Springer, Berlin, Heidelberg, Vol. 28, 2009, pp. 33-48.

[10] Rasheed, T. M., Reynaud, L., \& Al Agha, K. A stable clustering scheme for large scale mobile ad hoc networks. In 2005 IEEE/Sarnoff Symposium on Advances in Wired and Wireless Communication, 2005, pp. 121-124.

[11] Ahmad, M., Ikram, A. A., Wahid, I., Inam, M., Ayub, N., \& Ali, S. A bio-inspired clustering scheme in wireless sensor networks: BeeWSN. Procedia Computer Science, 130, 2018, pp. 206-213.

[12] Ye, Z., Tong, X., Yang, H., Meng, L., Xue, W., \& Wang, L. The Strip Clustering Scheme for data collection in large-scale Wireless Sensing Network of the road. International Journal of Pavement Research and Technology, 11(2), 2018, pp. 138-145.

[13] Rao, P. S., Jana, P. K., \& Banka, H. A particle swarm optimization-based energy efficient cluster head selection algorithm for wireless sensor networks. Wireless networks, 23(7), 2017, pp. 2005-2020.

[14] Rajagopal, A., Somasundaram, S., Sowmya, B., \& Cerna, P. Performance Analysis for Efficient Cluster Head Selection in Wireless Sensor Network Using RBFO and Hybrid BFO-BSO. International Journal of Wireless Communications and Mobile Computing, 6(1), 2018, pp. 1.

[15] Singh, S. S., Kumar, M., Saxena, R. \& Priya. Application of Particle Swarm Optimization for Energy Efficient Wireless Sensor Network: A Survey, International Journal of Engineering Science \& Advanced Technology, 2(5), 2002, pp. 1246 - 1250.

[16] Ali, S., Fathima, K., \& Sumitha, T. To Enhance the Lifetime of WSN Network using PSO. In Proceedings of International Conference on Global Innovations in Computing Technology (ICGICT'14), International Journal of Innovative Research in Computer and Communication Engineering, 2(1), 2014, pp. 1-6

[17] Clerc, $M$. The swarm and the queen: towards a deterministic and adaptive particle swarm optimization. In Proceedings of the IEEE Congress on Evolutionary Computation (CEC 99), Vol. 3, 1999.

[18] Ying, L., Haibin, Y., Peng, C. Application of PSO optimization based on clustering of wireless sensor network routing protocols. Journal of Control and Decision, 21(4), 2006, pp. 453-456.

[19] Kennedy, J. \& Eberhart, R. Swarm Intelligence, Morgan Kaufmann Publishers, 2001.

[20] Singh, B., \& Lobiyal, D. K. Energy-aware cluster head selection using particle swarm optimization and analysis of packet retransmissions in WSN. Procedia Technology, 4, 2012, pp. 171-176.

[21] Kumari, S., \& Shrivastava, M. Secure DSR protocol in MANET using energy efficient intrusion detection system. International Journal of Networks and Systems, 1(1), 2012, pp. 6-11. 
[22] Tillett, J., Rao, R., Sahin, F. Cluster-head identification in ad hoc sensor networks using particle swarm optimization, IEEE International Conference on Personal Wireless Communications, Delhi, India, 2002

[23] Eberhart, R. \& Shi, Y. Particle swarm optimization: developments, applications and resources. Proceedings of the IEEE Congress on Evolutionary Computation (IEEE Cat. No.01TH8546), 2001

[24] Ahmad, M., Ikram, A. A., Wahid, I., Inam, M., Ayub, N., Ali, S. A bio-inspired clustering scheme in wireless sensor networks: BeeWSN. Procedia Computer Science, 130, 2018, pp. 206-213

[25] Bali, V., Rathore R. S., Sirohi, A., Verma, P. A Framework to Provide a Bidirectional Abstraction of the Asymmetric Network to Routing Protocols. Second International Conference on Emerging Trends in Engineering \& Technology, 12/2009, pp. 1143-1150.

[26] Dong, Y., Tang, J., Xu, B., Wang, D. An application of swarm optimization to nonlinear programming. Computers and Mathematics with Applications, 49, 2005, pp. 1655-1668

[27] Haseeb, K., Abu Bakar, K., Ahmed, A., Darwish, T., Ahmed, I. WECRR: Weighted EnergyEfficient Clustering with Robust Routing for Wireless Sensor Networks. Wireless Personal Communications, 2017

[28] Kanchan, P., Shetty D Pushparaj. A quantum inspired PSO algorithm for energy efficient clustering in wireless sensor networks. Cogent Engineering, 5(1), 2018, pp. 1-16

[29] Popovic, G., Djukanovic, G., Kanellopoulos, D. Cluster Head Relocation Based on Selfish Herd Hypothesis for Prolonging the Life Span of Wireless Sensor Networks. Electronics, 7, 2018, pp. 1-15.

[30] Huang, Yourui, Qu, L., and Tang, C. Optimal Coverage Scheme based on QPSO in Wireless Sensor Networks. Journal of Networks, 7, 2012, pp. 1362-1368.

[31] Toor, A. S. \& Jain, A. K. A Novel Energy Efficient Routing Protocol EACBM for Scalable Wireless Sensor Networks. International Journal of Computer Network and Information Security, 5, 2018, pp. 9-17

[32] Guleria, K. \& Verma, A. K. Comprehensive review for energy efficient hierarchical routing protocols on wireless sensor networks. Wireless Networks, 2018, pp. 1-25

[33] Sasikumar, \& P., Khara, S. K-Means Clustering in Wireless Sensor networks. In Proceedings of Fourth International Conference on Computational Intelligence and Communication Networks, 2012, pp. 140-144

[34] Chandirasekaran, D. \& Jayabarathi, T. Cat swarm algorithm in wireless sensor networks for optimized cluster head selection: a real time approach. Cluster Computing, 2017, pp. 1-11 